\title{
Foreword: Special Issue on Database Theory
}

\author{
Balder ten Cate ${ }^{1} \cdot$ Wang-Chiew Tan ${ }^{2}$
}

This Theory of Computing Systems special issue contains five invited, extended journal articles of papers presented at the International Conference on Database Theory (ICDT 2013), which was held jointly with the International Conference on Extending Database Technology (EDBT 2013) on March 18 - 22, 2013 in Genoa, Italy. ICDT is one of the major conferences for database theory. The ICDT conference started in 1986 and was a biennial conference until 2009, when it became an annual conference and is jointly held with EDBT.

The submissions for the special issue underwent thorough journal reviewing and revision process, in accordance with the usual standards of Theory of Computing Systems. The papers in this special issue are (in no particular order):

- Fast Learning of Restricted Regular Expressions and DTDs, Dominik D. Freydenberger, Timo Kötzing

- Lower bounds on the error of query sets under the differentially-private matrix mechanism, Chao Li, Gerome Miklau

- Structural Tractability of Counting of Solutions to Conjunctive Queries, Arnaud Durand, Stefan Mengel

$\bowtie \quad$ Wang-Chiew Tan

tan@cs.ucsc.edu

Balder ten Cate

balder.tencate@gmail.com

1 LogicBlox and UC Santa Cruz, Santa Cruz, CA 95064 USA

2 University of California, Santa Cruz, CA, USA 
- Which XML schemas are streaming bounded repairable? Pierre Bourhis, Gabriele Puppis, Cristian Riveros

- XML Compression via Directed Acyclic Graphs, Mireille Bousquet-Melou, Markus Lohrey, Sebastian Maneth, Eric Noeth

We thank the authors and the reviewers for all their hard work, without which this special issue would not have been possible. 